\title{
Delayed post-traumatic syringohydromyelia after an uncomplicated fracture of the spine: Case report and literature review
}

\author{
F Güler-Uysal ${ }^{1}$, S Orkun ${ }^{2}$, A Cila $^{3}$ and N Özgirgin ${ }^{1}$ \\ ${ }^{1}$ Specialist of PMR, Ankara Rehabilitation Center, Ankara; ${ }^{2}$ Assoc. Prof. in PMR, Ankara Rehabilitation Center, \\ Ankara; ${ }^{3}$ Assoc. Prof. In Radiology, Dept. of Radiology, Hacettepe University, Faculty of Medicine, Ankara, Turkey
}

\begin{abstract}
A patient who had had a traffic accident resulting with a compression fracture of the L1 vertebra but with no neurological deficits, developed urinary incontinence and weakness in his lower extremities 25 years after the spinal injury. An MRI scan of the spine revealed gliosis of the conus medullaris at the level of the compression fracture, with syringohydromyelia extending from T6 to conus medullaris. The patient underwent a rehabilitation program at the end of which he was able to ambulate independently.
\end{abstract}

Keywords: Syringomyelia; syringohydromyelia; spinal cord trauma; spinal cord injuries

\section{Introduction}

Syringomyelia is a chronic disorder involving the spinal cord, characterized by the presence of longitudinally oriented cavities and gliosis. The term 'hydromyelia' is used to describe the appearance of dilatation of the central canal. Consequently, recent literature tends to unite both terms as 'syringohydromyelia' or use only syringomyelia in order to define the spectrum of this disease. ${ }^{1}$ The role of trauma in the pathogenesis of syringomyelia has been known for a long time. Post traumatic syringomyelia is the most common cause of neurological deterioration in patients who have sustained serious spinal cord trauma and who develop progressive neurological deterioration months or years after their injury. The overall incidence has been estimated to range from $1.3 \%$ to $3.2 \%$ of all spinal cord injuries (SCI), ${ }^{2,3}$ but recent evidence from magnetic resonance imaging (MRI) which is the most accurate modality for its diagnosis, ${ }^{1,4}$ has shown that the incidence is actually higher.

Although delayed post-traumatic syringomyelia in paraplegia or tetraplegia is a rather common entity, patients presenting with delayed post-traumatic syringomyelia several years after an uncomplicated spinal fracture with no neurological deficit is extremely rare and the significance of the past history of spinal trauma may sometimes be overlooked. ${ }^{5,6}$

We report a patient with syringohydromyelia presenting 25 years after a spinal injury that had caused a vertebral fracture but absolutely no neurological symptoms or signs, and draw attention to the subject.

\section{Case History}

A 62 year old male presented in May 1994 with a 12 month history of bilateral progressive leg weakness and urinary incontinence. Twenty-five years previously he had had a traffic accident which caused a crush fracture of $\mathrm{L} 1$ vertebra but with no transient or persistent neurological deficits. He had complete bed rest for one month, during which he could again not recall any neurological symptoms. He had had pain in the low back area lasting for a week after the accident and no other pain or weakness until 25 years post injury. In 1993, he began to develop progressive weakness of both the lower extremities accompanied by urinary incontinence. Evaluation by a neurosurgeon then revealed an incomplete paraplegia (Frankel D). A lumbar myelogram showed no block to the flow of contrast around the level of the old vertebral fracture. An MRI scan of the spine revealed a central cystic cavity of the spinal cord extending from T6 to conus medullaris, indicating a hydromyelia (Figure 1). T2W sagittal images demonstrated hyperintensity of conus medullaris consistent with cord injury and gliosis at the level of the old compression fracture (Figure 2). No craniovertebral junction abnormalities such as the Chiari malformation nor of arachnoiditis, which are common causes of syringomyelia, were observed on the MRI. There was no family history of any neurological disease. He also did not have a pre-existing clinical abnormality other than the Ll crush fracture that might be related to the development of the cavity within the spinal cord. Since the syrinx was too small in diameter, and extended through $\mathrm{T} 6$ to conus medullaris, neurosurgical intervention was not considered and he was referred to Ankara Rehabilitation Center for rehabilitation.

When the patient was referred to our center, he had lost motor power in the lower extremities within a 


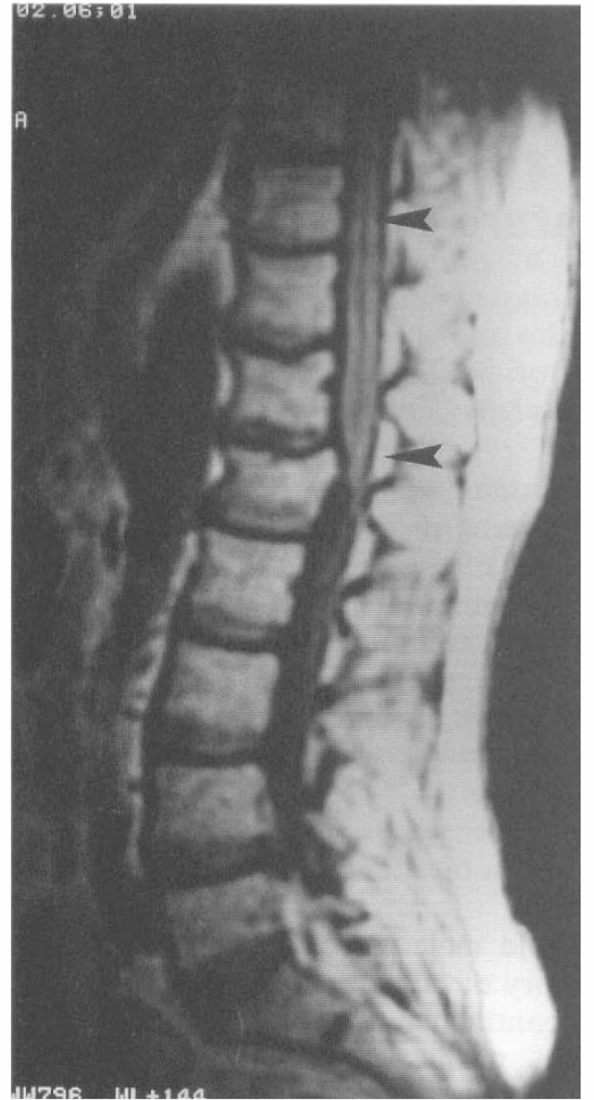

Figure 1 Midsagittal T1W SE image shows L1 compression with posterior subluxation compressing conus medullaris. Central syrinx cavity extending from conus to upper thoracal cord is seen as a thin black line (arrowheads)

period of months and was wheelchair bound. He had fairly good sitting balance but no standing balance. Cranial and upper extremity evaluation were completely normal. Wasting and weakness in the lower extremities were notable, the left side being worse than the right. The deep tendon reflexes in the lower extremities were abolished and the plantar responses could not be elicited. Pin prick, light touch, temperature sensation, joint position sense as well as sweating were diminished below T10 level. Urodynamic studies revealed detrusor hyperreflexia and external sphincter dyssynergia. Control MRI was performed and he was again evaluated by a neurosurgeon. Surgical intervention was not considered as there was no enlargement of the cyst cavity and no worsening of the neurological deficits.

Rehabilitation was first aimed to strengthen his abdominal and back muscles through mat exercises to maintain good posture and standing balance. He was then treated within the parallel bars for posture and balance with the aid of posterior shells. Meanwhile he was given anticholinergic medication for his bladder dysfunction. After succeeding within the parallel bars, he was prescribed bilateral KAFOs with pelvic belt

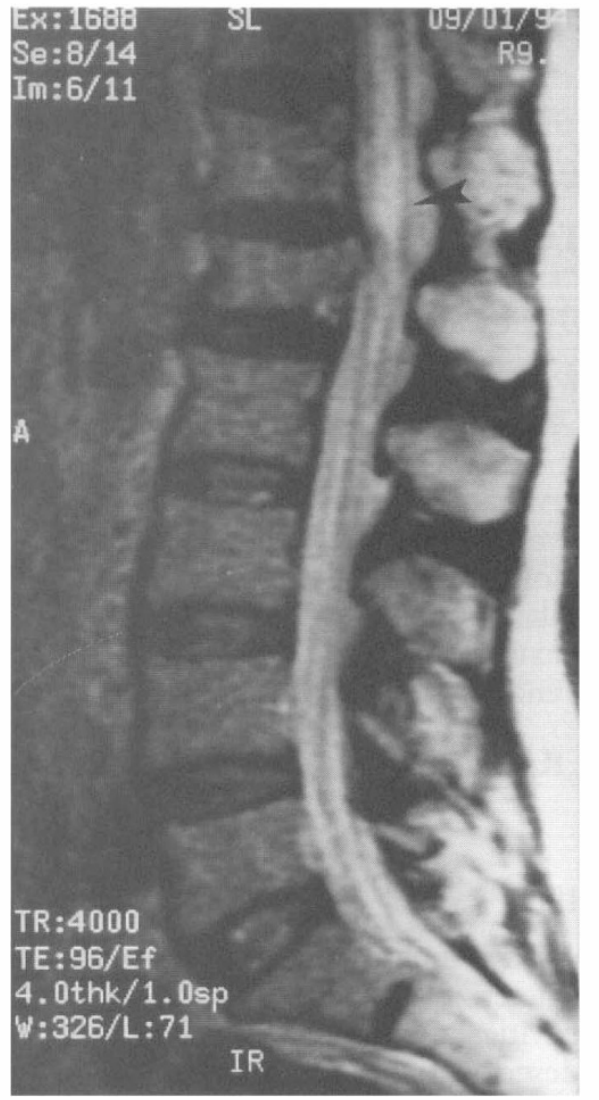

Figure 2 Midsagittal T2W SE image showing signal hyperintensity in conus medullaris (arrowhead) consistent with cord injury and gliosis

and crutches. He gained a 'swing to' gait within 4 months and performed clean intermittent self catheterization for urinary excretion at discharge.

\section{Discussion}

Post-traumatic syringomyelia is a well recognized late sequela to spinal trauma in spinal cord patients. The clinical presentation is usually marked by pain, ascending sensory loss, increased muscle weakness and depressed deep tendon reflexes in paraplegic or tetraplegic patients. Dissociated sensory loss, with loss of pain and temperature sensation and preservation of light touch and proprioception is common. ${ }^{5,7}$ Although post-traumatic syringomyelia in paraplegic and tetraplegic patients has been known for a long time, syringomyelia occurring as a result of spinal injury in patients with no residual neurological deficit is a rather new entity, and the history of previous spinal trauma can easily be overlooked.

Diagnostic studies show post-traumatic syrinxes to originate at the level of the initial spinal cord trauma, to be most frequently located in the dorsal region of the spinal cord, and to be variable in length, width and 
position throughout the cord. Most often they extend proximally from the level of injury, ${ }^{3,5}$ just like our case in which the caudal end of the syrinx cavity corresponded to the level of the previous fracture (Figure 1). The relationship between the syrinx cavity and the vertebral fracture, the presence of gliosis of the conus medullaris indicating some previous intrinsic cord damage (Figure 2), together with the absence of Chiari malformations, arachnoiditis or tethered cord determination by MRI, strongly suggests the role of previous spinal injury in the pathogenesis of syringohydromyelia in our patient.

Barnett ${ }^{8}$ defined three categories of post-traumatic syringomyelia: (1) following traumatic paraplegia and tetraplegia, (2) following minor or moderate spinal cord injury and (3) as a late complication of spinal trauma producing adhesive arachnoiditis. Four types of trauma were suggested by Van den Bergh, ${ }^{6}$ to be responsible for the development of syringomyelia: repeated microtrauma, trauma followed by arachnoiditis, severe single trauma followed by persistent para or tetraplegia and minor single trauma followed by reversible paraplegia. Our patient had a spinal trauma which was severe enough to cause a crush fracture of the L1 vertebra. But this trauma caused only minor cord damage to the conus medullaris with no immediate neurological deficits but induced the development of syringohydromyelia during the following 25 years.

Barnett and Jousse ${ }^{9}$ reviewed six cases that they felt were sufficiently well documented to be accepted as examples of post-traumatic syringomyelia following mild to moderate trauma. Two of their patients had no neurological symptoms following the original spinal injury whereas the remaining four had transient or persistent mild sensory or motor symptoms. Although there have been more recently published series of post-traumatic syringomyelia, no other cases with uncomplicated spinal fracture has been defined until Bleasel's report of two cases in 1991, one of which had transient neurological symptoms post-injury and the other with no neurological symptoms after a crush fracture of the L1 vertebra. ${ }^{5}$ Our case is the fourth report of delayed post-traumatic syringohydromyelia in the literature with no immediate neurological signs post-injury.

The interval between the original injury and the later presentation with syringomyelia varies between these reports of cases. A range of three months to 34 years exists in the literature for paraplegia and tetraplegia and 4 to 22 years for the mild to moderate spinal injury patients.9 Bleasel's case developed a syrinx cavity after 41 years post-injury which is up to now the latest one in the literature. ${ }^{5}$ Our patient had an interval of 25 years between the trauma and the start of neurological deficits caused by the syrinx formation.

Clinicopathological studies have resulted in the concept of a spectrum of post-traumatic spinal cord pathology from arachnoiditis to microcystic degen- eration and syringohydromyelia. ${ }^{10} \mathrm{~A}$ syrinx may develop after spinal cord trauma, either soon after resorption of an intramedullary hematoma or as a delayed phenomenon after cord contusion or compression with microcystic cavitation. ${ }^{6,11}$ Deficient or altered blood supply in the region of trauma could also be blamed for the production of the cavity within the cord. ${ }^{12}$ Microinfarcts as well as lysosomal and other cellular enzymes may play an important role in the liquefaction process and cyst formation. ${ }^{7}$ Cores of necrotic tissue may extend for several segments from the level of injury and these could liquefy and form a cavity. ${ }^{7,10,13}$ Cyst extension, leading to the development of neurological symptoms and signs, has been thought to be due to the transmission to the cyst cavity of cerebrospinal fluid pressure fluctuations occurring with flexion and extension of the spine and straining. Any local arachnoid adhesions tethering the spinal cord enhance the transmission of the cerebrospinal fluid pressure fluctuations and cyst extension and may contribute to cord ischaemia. ${ }^{5,7}$ In our patient, we hypothesize that there must have been minor cord damage resulting with no neurological deficit at the time of trauma and the enlargement of the central canal would be due to the compression effect of the fractured vertebra on conus medullaris. Castillo et al ${ }^{14}$ propose that syringohydromyelia may occur as a consequence of an extramedullarly compressive lesion, possibly owing to ischaemia of the cord with secondary degeneration of the gray matter or by filling of the central canal by extrachoroidally produced cerebrospinal fluid (CSF). Extrinsic compression may lead to neuroglial damage with enlargement of extracellular perivascular spaces. The traumatic gliosis and the stenosis of the dural sac in the region may alter the CSF dynamics so that abnormal entry of CSF into the cord via the enlarged Virchow-Robin spaces dissects the cord and results in syringohydromyelia which enlarges and propagates due to a water-hammer effect.

Surgery, especially syringoperitoneal shunting, is most likely to be the useful treatment if there is progressive neurological deficit, and should be avoided if the patient's condition is not deteriorating, and when there is an extensive syringomyelia involving most of the spinal cord. ${ }^{5,6}$ Surgical intervention was not considered for our patient because he had an extensive syrinx cavity which was very small in diameter and the neurological deficits did not show any deterioration at follow up studies.

The development of a syrinx cavity after a vertebral fracture causing no neurological deficit is a very rare condition. Patients presenting with syringomyelia must be evaluated for a previous history of minor or moderate spinal trauma that might have occurred many years ago, and vice versa, patients who experience a vertebral fracture with no neurological deficits should be followed up for a syrinx formation even after some decades post-injury. 


\section{References}

1 Sherman JL, Barkovich AJ, Citrin CM. The MR appearance of syringomyelia: New observations. AJR 1987; 148: $381-391$.

2 Umbach I, Heilporn A. Review article: Post-spinal cord injury syringomyelia. Paraplegia 1991; 29: $219-221$.

3 Doyle WK, Wilmot C, Hall KM et al. Trauma of the spine and spinal cord. In: Joynt RJ, ed. Clinical Neurology. Vol 3 (Chapter 47). JB Lippincott: Philadelphia, 1992; $1-58$.

4 Nidecker A, Kocher M, Maeder M et al. MR-imaging of chronic spinal cord injury. Association with neurologic function. Neurosurg Rev 1991; 14: $169-179$.

5 Bleasel A, Clouston P, Dorsch N. Post-traumatic syringomyelia following uncomplicated spinal fracture. J Neurol Neurosurg Psychiatry 1991; 54: 551-553.

6 Van den Bergh R. Pathogenesis and treatment of delayed posttraumatic syringomyelia. Acta Neurochir (Wien) 1991; 110: 82 86.

7 Biyani A, El Masry WS. Post-traumatic syringomyelia: a review of the literature. Paraplegia 1994; 32: $723-731$.

8 Barnett HJM. Trauma and syringomyelia. In: Barnett HJM, Foster JB, Hudgson P, eds. Syringomyelia. WB Saunders: London, 1973; 127-128.
9 Barnett HJM, Jousse AT. Posttraumatic syringomyelia (cyctic myelopathy). In: Vinken PJ, Bruyn GW, eds. Handbook of clinical neurology, Vol 26. North-Holland: Amsterdam, 1976; $113-157$.

10 Macdonald RL, Findlay JM, Tator CH. Microcystic spinal cord degeneration causing posttraumatic myelopathy. J Neurosurg 1988; 68: 466 - 471 .

11 Kiewitchz A. Spinal cord diseases. In: Rowland LP, ed. Merrit's Textbook of Neurology, 9th ed. Williams and Wilkins: Philadelphia, 1995; 737-753.

12 Finlayson AI. Syringomyelia and related conditions. In: Joyn RJ, ed. Clinical Neurology, Vol 3 (Chapter 45). JB Lippincott Philadelphia, 1992; 1-17.

13 Kerslake RW, Jaspan T, Worthington BS. Magnetic resonance imaging of spinal trauma. Br J Radiology 1991; 64: 386-402.

14 Castillo M, Quencer RM, Green BA, Montalvo BM. Syringomyelia as a consequence of compressive extramedullary lesions: postoperative clinical and radiological manifestations. $A J R$ 1988; 150: $391-396$. 